\title{
NOTE ON TRANSCRIPTION
}

The transcription system of A Dictionary of Egyptian Arabic, by Badawi and Hinds, has been used with minor changes. The long vowels ii, uu, aa, etc. have been replaced by $\hat{\imath}, \hat{u}, \hat{a}$, etc., and the $\mathrm{x}$ has been replaced by $\mathrm{kh}$. The more common transliteration is used for the Arabic consonants $\varepsilon, \mathrm{y}$, and $\int$-that is, ', gh, and sh, respectively. In quotations, the transcription system of the quoted author is followed. Some place names or words familiar to the reader, such as Cairo, Alexandria, and sheikh, have been written with their anglicized spellings; otherwise the strict transcription is used. In general, I have added an $s$ to singular Arabic words to form an anglicized plural, with the exception of a few frequently employed terms. I mainly use the colloquial Egyptian Arabic, with the exception of official names-for instance, of newspapers-which are transcribed in standard Arabic. 
THIS PAGE INTENTIONALLY LEFT BLANK 


$$
-
$$


THIS PAGE INTENTIONALLY LEFT BLANK 